\$24. The perpendiculars from the vertices on the opposite sides of a triangle bisect the angles of the triangle formed by joining the feet of the perpendiculars. (Proof by means of the polar figure).

Let $\mathrm{ABC}$ be the polar triangle, $L, M, N$, the poles of the perpendiculars in the original triangle, i.e., $L$ is a point in $B C$ such that LA is a quadrant, \&c. Then DEF is the polar of the triangle formed by joining the feet of the perpendiculars in the original triangle; and it is required to show that $\mathrm{L}, \mathrm{M}, \mathrm{N}$ bisect the sides of DEF externally.

$\mathrm{L}, \mathrm{M}, \mathrm{N}$ are the poles of the perpendiculars of $\mathrm{ABC}$;

$\therefore \mathrm{A}, \mathrm{B}, \mathrm{C}$ are the middle points of the sides of DEF ( $(22)$.

$\therefore \mathrm{L}, \mathrm{M}, \mathrm{N}$ bisect the sides externally.

Although not strictly within the scope of this paper, the following proof of the theorem of $\$ 23$ may be interesting.

Let $\mathrm{ABCD}$ (fig. 10) be the quadrilateral, $\mathrm{AC}$ and $\mathrm{BD}$ being quadrants. Then (AGCK) $=-1$, and $A C$ is a quadrant; $\therefore G C=C K$. Similarly, $\mathbf{G B}=\mathbf{B L}$.

Now, in the triangle LGK, B bisects LG internally, and A bisects GK externally ; $\because \mathrm{E}$ bisects LK. And from triangle GLK $\mathrm{F}$ bisects LK externally ; $\therefore$ EF is a quadrant.

\title{
Note on the Condensation of a Special Continuant.
}

\section{By Thouas Murr, M.A., F.R.S.E.}

[Held over from Third Meeting.]

\$ 1. The continuant referred to is that in which the elements of the main diagonal are all equal (to $x$, say), the elements of the one minor diagonal all equal (to $b$, say), and the elements of the other minor diagonal all equal (to $c$, say). It may be denoted by $\mathbf{F}(b, x, c, n)$ when it is of the $n$th order. Professor Wolstenholme has recently given two elegant theorems regarding the condensation of $F(1, x, 1, n)$. I wish to establish the analogous theorems for $\mathrm{F}(b, x, c, n)$.

$\$ 2$. It may be necessary to premise that a determinant whose elements are all zeros, except those in the main diagonal and in the two diagonals drawn through the places $(1,3),(3,1)$ parallel to the main diagonal, is expressible as the product of two continuants. Thus 


$$
\begin{aligned}
& \left|\begin{array}{ccccccc}
a_{1} & 0 & c_{2} & 0 & 0 & 0 & 0 \\
0 & a_{2} & 0 & c_{2} & 0 & 0 & 0 \\
b_{1} & 0 & a_{3} & 0 & c_{3} & 0 & 0 \\
0 & b_{2} & 0 & a_{4} & 0 & c_{4} & 0 \\
0 & 0 & b_{3} & 0 & a_{5} & 0 & c_{3} \\
0 & 0 & 0 & b_{4} & 0 & a_{6} & 0 \\
0 & 0 & 0 & 0 & b_{5} & 0 & a_{7}
\end{array}\right| \text { or } \mathrm{D}_{7} \\
& =\left|\begin{array}{cccc}
a_{1} & c_{1} & 0 & 0 \\
b_{1} & a_{3} & c_{3} & 0 \\
0 & b_{3} & a_{5} & c_{5} \\
0 & 0 & b_{5} & a_{7}
\end{array}\right| \quad\left|\begin{array}{ccc}
a_{2} & c_{2} & 0 \\
b_{2} & a_{4} & c_{4} \\
0 & b_{4} & a_{6}
\end{array}\right|
\end{aligned}
$$

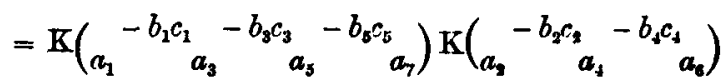

and similarly--

$$
\begin{aligned}
& \mathrm{D}_{6}=\left|\begin{array}{ccc}
a_{1} & c_{1} & 0 \\
b_{1} & a_{3} & c_{3} \\
0 & b_{3} & a_{5}
\end{array}\right| \cdot\left|\begin{array}{ccc}
a_{2} & c_{3} & 0 \\
b_{3} & a_{4} & c_{4} \\
0 & b_{4} & a_{6}
\end{array}\right|, \\
= & \mathrm{K}\left(\begin{array}{ccc}
-b_{1} c_{1} & -b_{3} c_{3} \\
a_{1} & a_{3} & a_{5}
\end{array}\right) \cdot \mathrm{K}\left(\begin{array}{ccc}
-b_{2} c_{2} & -b_{4} c_{4} \\
a_{3} & a_{4} & a_{8}
\end{array}\right)
\end{aligned}
$$

and therefore, as we may observe in passing,

$$
\frac{\mathrm{D}_{7}}{\mathrm{D}_{6}}=a_{7}-\frac{b_{5} c_{3}}{a_{5}}-\frac{b_{3} c_{3}}{a_{3}}-\frac{b_{1} c_{1}}{a_{1}}
$$

§3. Also, we may note that since

$$
\begin{aligned}
& \mathrm{K}\left(a+b \quad a^{-b^{2}}-b^{2}-b^{2}-b^{2} a\right)=
\end{aligned}
$$

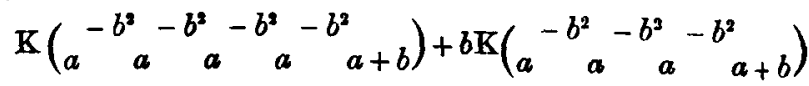

and since the first term in the right-hand member equals

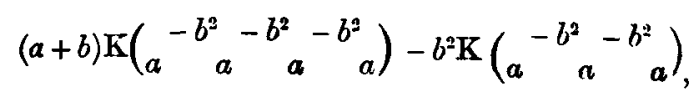

and the second term equals

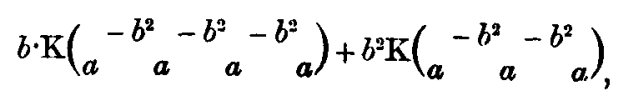


we have the identity in continuants

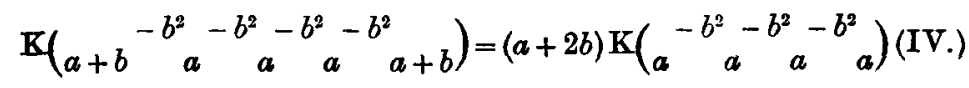

$\S 4$. Now, taking the case of $\mathrm{F}(b, x, c, n)$ where $n$ is odd, we have

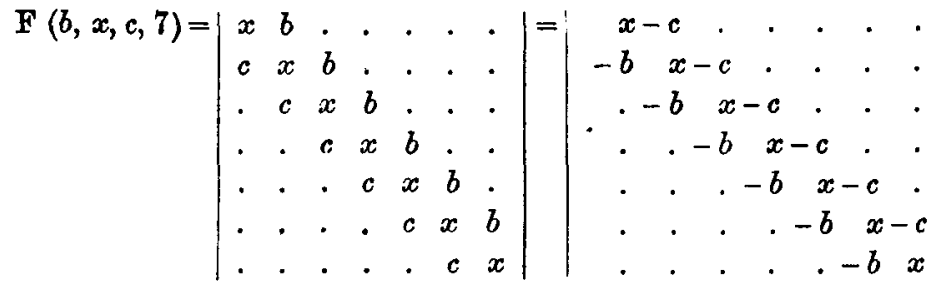

and therefore by multiplication

$$
\begin{aligned}
& {[\mathrm{F}(b, x, c, 7)]^{2}=\left|\begin{array}{ccccccc}
x^{2}-b c & 0 & -b^{2} & . & . & . & \cdot \\
0 & x^{2}-2 b c & 0 & -b^{2} & \cdot & . & \cdot \\
-c^{2} & 0 & x^{2}-2 b c & 0 & -b^{2} & . & \cdot \\
\cdot & -c^{2} & 0 & x^{2}-2 b c & 0 & -b^{2} & \cdot \\
\cdot & . & -c^{2} & 0 & x^{2}-2 b c & 0 & -b^{2} \\
\cdot & . & . & -c^{2} & 0 & x^{2}-2 b c & 0 \\
. & . & . & . & -c^{2} & 0 & x^{2}-b c
\end{array}\right|} \\
& =\left|\begin{array}{cccc}
x^{2}-b c & -b^{2} & \cdot & \cdot \\
-c^{2} & x^{2}-2 b c & -b^{2} & \cdot \\
\cdot & -c^{2} & x^{2}-2 b c & -b^{2} \\
\cdot & \cdot & -c^{2} & x^{2}-b c
\end{array}\right|\left|\begin{array}{ccc}
x^{2}-2 b c & -b^{2} & \cdot \\
-c^{2} & x^{2}-2 b c & -b^{2} \\
\cdot & -c^{2} & x^{2}-2 b c
\end{array}\right| \text { by } \S 2 .
\end{aligned}
$$

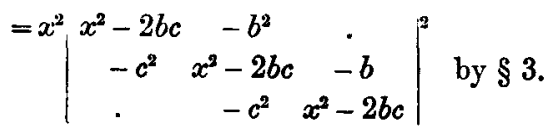

and consequently we have

$$
\mathbf{F}(b, x, c, 7)=x \mathbf{F}\left(b^{2}, x^{2}-2 b c, c^{3}, 3\right),
$$

the general theorem evidently being

$$
\mathbf{F}(b, x, c, 2 n+1)=x \mathbf{F}\left(b^{2}, x^{2}-2 b c, c^{2}, n\right)
$$

In exactly the same way we find the complementary theorem

$$
\mathrm{F}(b, x, c, 2 n)=\mathrm{F}\left(b^{2}, x^{2}-2 b c, c^{2}, n\right)+b c \mathrm{~F}\left(b^{2}, x^{3}-2 b c, c^{2}, n-1\right) \ldots
$$

BegchCroft, Bishoptor,

2nd Jan. 1884. 